\title{
Phylogenetics of Hydroidolina (Hydrozoa: Cnidaria)
}

\author{
PAULYN CARTWRIGHT ${ }^{1}$, NATHANIEL M. EVANS ${ }^{1}$, CASEY W. DUNN ${ }^{2}$, ANTONIO C. MARQUES $^{3}$, \\ MARIA PIA MIGLIETTA ${ }^{4}$, PETER SCHUCHERT ${ }^{5}$ AND ALLEN G. COLLINS ${ }^{6}$
}

${ }^{1}$ Department of Ecology and Evolutionary Biology, University of Kansas, Lawrence, KS 66049, USA, ${ }^{2}$ Department of Ecology and Evolutionary Biology, Brown University, Providence RI 02912, USA, ${ }^{3}$ Departamento de Zoologia, Instituto de Biociências, Universidade de São Paulo, São Paulo, SP, Brazil, ${ }^{4}$ Department of Biology, Pennsylvania State University, University Park, PA 16802, USA, ${ }^{5}$ Muséum d'Histoire Naturelle, CH-1211, Genève, Switzerland, ${ }^{6}$ National Systematics Laboratory of NOAA Fisheries Service, $\mathrm{NMNH}$, Smithsonian Institution, Washington, DC 20013, USA

\begin{abstract}
Hydroidolina is a group of hydrozoans that includes Anthoathecata, Leptothecata and Siphonophorae. Previous phylogenetic analyses show strong support for Hydroidolina monophyly, but the relationships between and within its subgroups remain uncertain. In an effort to further clarify hydroidolinan relationships, we performed phylogenetic analyses on 97 hydroidolinan taxa, using DNA sequences from partial mitochondrial $16 \mathrm{~S}$ rDNA, nearly complete nuclear $18 \mathrm{~S}$ rDNA and nearly complete nuclear $28 S$ rDNA. Our findings are consistent with previous analyses that support monophyly of Siphonophorae and Leptothecata and do not support monophyly of Anthoathecata nor its component subgroups, Filifera and Capitata. Instead, within Anthoathecata, we find support for four separate filiferan clades and two separate capitate clades (Aplanulata and Capitata sensu stricto). Our data however, lack any substantive support for discerning relationships between these eight distinct hydroidolinan clades.
\end{abstract}

Keywords: phylogenetics, Hydroidolina, Hydrozoa, Cnidaria

Submitted 30 November 2007; accepted 12 May 2008; first published online 29 July 2008

\section{INTRDDUCTION}

Hydroidolina (=Leptolina) is a clade of hydrozoans comprising Leptothecata (=Leptomedusae, Thecata), Anthoathecata (=Anthomedusae, Athecata) and Siphonophorae (Collins, 2002; Marques \& Collins, 2004; Collins et al., 2006). Amongst the approximately 3220 valid species of Hydroidolina (Bouillon et al., 2006), there exist vast amounts of diversity in the morphologies of hydroids and medusae as well as in life cycles. Uncovering a robust phylogeny for Hydroidolina would shed insight into the patterns underlying this diversity and provide a framework for generating hypotheses concerning processes responsible for their evolution. In addition, molecular phylogenies of Hydroidolina could help serve as a guide to taxonomic classification, which has been somewhat problematic, in large part due to inconsistencies in classifications of hydroids and medusae (e.g. Bouillon, 1985, 1994).

Hydrozoan phylogenetics has seen much progress in recent years, particularly in revealing major hydrozoan lineages and questioning others. For example, phylogenetic analyses have shown that Hydrozoa comprises two well-supported, reciprocally monophyletic clades, Trachylina and Hydroidolina (Marques \& Collins, 2004; Marques, 2001a; Collins, 2002; Collins et al., 2006; Van Iten et al., 2006). Siphonophorae is a clade (Collins, 2002; Dunn et al., 2005), but its phylogenetic

Corresponding author:

P. Cartwright

Email: pcart@ku.edu position within Hydroidolina is uncertain (Collins, 2002; Collins et al., 2006). Similarly, there is strong support for the monophyly of Leptothecata (Collins et al., 2006; Leclère et al., 2007), but no well-supported hypotheses have emerged regarding its relationship with other hydroidolinans. Molecular phylogenetic studies do not support the monophyly of Anthoathecata and instead suggest that it is a paraphyletic assemblage that has given rise to one or more hydroidolinan groups (Marques \& Collins, 2000; Marques, 2001a; Collins, 2002; Collins et al., 2006; Van Iten et al., 2006).

Although these studies illuminated the phyletic status of the three main groups of Hydroidolina, the relationships within and between these groups remain uncertain (Collins et al., 2006). In addition, with exception to studies on Kirchenpaueriidae (Peña Cantero \& Marques, 1999), Corynidae (Collins et al., 2005), Siphonophorae (Dunn et al., 2005), Tubulariidae (Marques \& Migotto, 2001), Campanulariidae (Govindarajan et al., 2006), HebellidaeLafoeidae (Marques et al., 2006) and Plumularioidea (Leclère et al., 2006), relationships within component hydroidolinan groups have not been studied within a detailed phylogenetic framework. In an effort to further clarify relationships within Hydroidolina, we greatly augmented the published molecular dataset of hydroidolinan taxa using three molecular markers, the nuclear large (28S) and small (18S) subunit rDNAs and the mitochondrial large subunit rDNA (16S). We present combined phylogenetic analyses of 97 hydroidolinan taxa (plus 13 trachyline taxa as outgroups) under maximum likelihood (ML) and parsimony (MP) criteria. The augmented dataset reveals new evolutionary 
patterns in morphology, although a more thorough sampling is needed to further clarify these patterns. These data suggest that a molecular phylogenetic approach is promising for guiding future taxonomic classifications but further study is needed to elucidate phylogenetic patterns of the deeper nodes within Hydroidolina.

\section{MATERIALS AND METHODS}

\section{Taxa sampled, DNA isolation, amplification and sequencing}

The 110 hydrozoan taxa used in this study are arranged taxonomically in Table 1, including GenBank accession and museum voucher numbers. The sequences in Table 1 comprise both published and new DNA sequences generated for this study. Although most new sequences correspond to museum voucher specimens, some were included that had no associated vouchers, but for which published sequences of other markers were generated from the same DNA pool. For new sequences, genomic DNA was extracted using Qiagen DNeasy kits according to the manufacturer's protocol (QIAGEN Inc., Mississauga, ON) or a standard phenol/ chloroform protocol. The latter method involved tissue digestion with proteinase $\mathrm{K}(20 \mathrm{mg} / \mathrm{ml})$ in a lysis buffer $(20 \mathrm{mM}$ Tris-CL pH 8.0, 5 mM EDTA pH 8.0, $400 \mathrm{mM} \mathrm{NaCl}, 2 \%$ SDS), extraction with phenol/chloroform (1:1), precipitation with 2.5 vol. $95 \%$ EtOH and elution in TE or $\mathrm{H}_{2} \mathrm{O}$.

An approximately $600 \mathrm{bp}$ fragment of $16 \mathrm{~S}$ was amplified using a modified forward primer (F1Mod: TCGACTGTTTA CCAAAAACATA) and reverse primer (R2: ACGGAATGA ACTCAAATCATGTAAG) from Cunningham \& Buss (1993). Amplifications of 16S were conducted with the following thermal profile: 5 minutes (min.) at $94^{\circ} \mathrm{C} ; 5$ cycles of 50 seconds(s) at $94^{\circ} \mathrm{C}, 50 \mathrm{~s}$ at $45^{\circ} \mathrm{C}$ and $1 \mathrm{~min}$. at $72^{\circ} \mathrm{C} ; 30$ cycles of $5 \mathrm{~s}$ at $94^{\circ} \mathrm{C}, 50 \mathrm{~s}$ at $50^{\circ} \mathrm{C}$ and $1 \mathrm{~min}$. at $72^{\circ} \mathrm{C}$; $10 \mathrm{~min}$. at $72^{\circ} \mathrm{C}$. An approximately $1.8 \mathrm{~kb}$ portion of the gene coding for $18 \mathrm{~S}$ was amplified with universal eukaryotic primers as described by Medlin et al. (1988). Nearly complete, an approximately $3 \mathrm{~kb}$ portion of the gene coding for $28 \mathrm{~S}$ was amplified and sequenced according to Evans et al. (2008).

All gene fragments were purified and sequenced by Cogenics, Inc. (Houston, TX) and assembled and edited using Sequencher v4.5 (Gene Code Co., 2005). Sequences for each marker were aligned using the program MUSCLE (Edgar, 2004). Regions containing alignment ambiguities were removed using Gblocks vo.91b (Castresana, 2000) with default parameters except the minimum length block was set to 5 and half the taxa were allowed to be gaps for any given position (Table 2 ). The three datasets were concatenated into one combined dataset.

\section{Phylogenetic analysis}

Phylogenetic analyses were performed on individual markers and on the combined dataset using both maximum likelihood (ML) and parsimony (MP) criteria. ML searches were performed using GARLI vo.951.OsX-GUI (Zwickl, 2006) under an assumed GTR + I + G model with rates estimated from the data. The assumed model of nucleotide substitution was selected by using the Akaike information criterion (AIC) as implemented in ModelTest (Posada \& Crandall, 2000). For the combined dataset the ML analysis was repeated 10 times from random starting trees using default termination conditions. Each run gave identical topologies and similar likelihood scores. 100 bootstrap replications were run in GARLI vo.951.osX-GUI (Zwickl, 2006) under the same parameters.

MP analyses were performed using PAUP* 4.0.ob10 (Swofford, 1998). Heuristic analyses were run using 500 random addition sequences and TBR branch swapping. 100 bootstrap replications were run using 10 random addition sequences per replicate and TBR branch swapping. Most parsimonious trees were summarized as a strict consensus.

The concatenated, Gblocked DNA alignment and corresponding trees can be found in TreeBASE (http://www.tree base.org/treebase/index.html, accession No. S2066).

\section{RESULTS AND DISCUSSIDN}

After excluding the ambiguously aligned regions, the combined dataset of nearly complete $28 \mathrm{~S}$ rDNA, nearly complete $18 \mathrm{~S}$ rDNA and partial 16S rDNA contained 5046 characters, 1699 of which are parsimony informative. Information about individual markers is shown in Table 2. The markers were analysed separately under a ML optimality criterion and in a combined dataset under ML and MP optimality criteria. There is incongruence in topologies between the individual markers and very little support for most of the nodes in the $16 \mathrm{~S}$ and $18 \mathrm{~S}$ datasets (not shown). By contrast, the $28 \mathrm{~S}$ topology is almost identical to the combined dataset (not shown) but the combined dataset shows a higher frequency of well-supported nodes (bootstrap values $>50 \%$ ), than the $28 \mathrm{~S}$ topology (not shown). Given that the $28 \mathrm{~S}$ and combined dataset are congruent but the combined dataset gives better overall support values, we concluded that the combined dataset provides the most robust hypotheses. Thus, all subsequent discussions are confined to the analyses of the combined dataset (Figures 1 \& 2).

MP and ML analyses both support the monophyly of Hydroidolina (Figures 1 \& 2 ). Trachyline relationships are treated in detail in this volume (see Collins et al.) and are therefore not discussed here. The hydroidolinan taxa included in these analyses sort out into eight different monophyletic clades (Figures 1 \& 2; Table 1). The composition of taxa in these clades is identical in the ML and MP analyses (Figures 1 \& 2). Both optimality criteria support the monophyly of Leptothecata and Siphonophorae. In the ML and MP topologies, 'Anthoathecata' is a polyphyletic assemblage with leptothecates and siphonophores derived within anthoathecate lineages. Although it should be noted that all of the nodes separating the different anthoathecate lineages are weakly supported, consistent with previous phylogenetic analyses (Collins, 2002; Marques \& Collins, 2004; Collins et al., 2005, 2006; Dunn et al., 2005; Van Iten et al., 2006; Leclère et al., 2007). The separate anthoathecate clades that emerge from both the ML and MP analyses are Aplanulata (Collins et al., 2005), Capitata sensu stricto and four filiferan clades (Figures $1 \& 2$; Table 1). The composition and relationships within these major clades are discussed below.

Relationships among these major clades of Hydroidolina are uncertain. There is very little bootstrap support $(<50 \%)$ in the deeper nodes under both optimality criteria and there is incongruence in the ML and MP topologies between the 
Table 1. Taxon and sequence list. A complete list of sequences used in the analyses with GenBank accession numbers and museum voucher numbers. Those in bold represent new sequences generated for this study.

\begin{tabular}{|c|c|c|c|c|c|}
\hline Taxonomic hierarchy & & $28 S$ & $18 S$ & $16 S$ & Voucher \\
\hline \multicolumn{6}{|l|}{ Anthoathecata } \\
\hline \multicolumn{6}{|l|}{ Capitata } \\
\hline Cladocorynidae & Cladocoryne floccosa & $\mathrm{EU}_{272551}$ & $\mathrm{EU}_{272608}$ & $\mathrm{AY}_{512535}$ & \\
\hline Corynidae & Dipurena ophiogaster & $\mathrm{EU}_{272560}$ & $\mathrm{EU}_{272615}$ & $\mathrm{EU}_{305473}$ & KUNHM 2803 \\
\hline Corynidae & Sarsia nipponica & $\mathrm{EU}_{305530}$ & $\mathrm{EU}_{448096}$ & $\mathrm{EU}_{448100}$ & KUNHM 2627 \\
\hline Moerisiidae & Moerisia sp. & AY920801 & $\mathrm{AF}_{358083}$ & $\mathrm{AY}_{512534}$ & \\
\hline Pennariidae & Pennaria disticha & $\mathrm{EU}_{272581}$ & AY920762 & AMo88481 & \\
\hline Polyorchidae & Scrippsia pacifica & AY920804 & $\mathrm{AF}_{358091}$ & $\mathrm{AY}_{512551}$ & \\
\hline Porpitidae & Porpita sp. & AY920803 & $\mathrm{AF}_{358086}$ & $\mathrm{AY}_{512529}$ & \\
\hline Porpitidae & Velella sp./V. velella & $\mathrm{EU}_{272597}$ & $\mathrm{AF}_{358087}$ & $\mathrm{EU}_{305487}$ & \\
\hline Solanderiidae & Solanderia ericopsis & $\mathrm{EU}_{272593}$ & $\mathrm{EU}_{272636}$ & $\mathrm{AY} 78788$ & MHNG INVE 29593 \\
\hline Solanderiidae & Solanderia secunda & $\mathrm{EU}_{305533}$ & $\mathrm{EU}_{305502}$ & $\mathrm{EU}_{305484}$ & KUNHM 2611 \\
\hline Zancleidae & Zanclea prolifera & $\mathrm{EU}_{272598}$ & $\mathrm{EU}_{272639}$ & $\mathrm{EU}_{305488}$ & KUNHM 2793 \\
\hline \multicolumn{6}{|l|}{ Aplanulata } \\
\hline Candelabridae & Candelabrum cocksii & AY920796 & AY920758 & AY 512520 & MHNG INVE 29591 \\
\hline Corymorphidae & Corymorpha pendula & $\mathrm{EU}_{305510}$ & $\mathrm{EU}_{305494}$ & EU448098 & KUNHM 2962 \\
\hline Corymorphidae & Euphysora bigelowi & $\mathrm{EU}_{272563}$ & $\mathrm{EU}_{272618}$ & EU448099 & KUNHM 2829 \\
\hline Hydridae & Hydra circumcincta & AYo26371 & $\mathrm{AF}_{358080}$ & $\mathrm{AY}_{512521}$ & \\
\hline Tubulariidae & Ectopleura dumortieri & $\mathrm{EU}_{272561}$ & $\mathrm{EU}_{272616}$ & $\mathrm{EU}_{305474}$ & \\
\hline Tubulariidae & Ralpharia gorgoniae & $\mathrm{EU}_{272590}$ & $\mathrm{EU}_{272633}$ & $\mathrm{EU}_{305482}$ & KUNHM 2778 \\
\hline Tubulariidae & Zyzzyzus calderi & EU272599 & $\mathrm{EU}_{272640}$ & $\mathrm{EU}_{305489}$ & KUNHM 2777 \\
\hline \multicolumn{6}{|l|}{ Filifera I } \\
\hline Eudendriidae & Eudendrium californicum & $\mathrm{EU}_{305513}$ & $\mathrm{EU}_{305492}$ & $\mathrm{EU}_{305475}$ & KUNHM 2850 \\
\hline Eudendriidae & Eudendrium capillare & $\mathrm{EU}_{305514}$ & & $\mathrm{EU}_{305476}$ & KUNHM 2625 \\
\hline Eudendriidae & Eudendrium racemosum & $\mathrm{EU}_{272562}$ & $\mathrm{EU}_{272617}$ & $\mathrm{AY}_{787896}$ & \\
\hline \multicolumn{6}{|l|}{ Filifera II } \\
\hline incertae sedis & Brinckmannia hexactinellidophila & $\mathrm{EU}_{272550}$ & $\mathrm{EU}_{272607}$ & AM183123 & MHNG INVE 38148 \\
\hline Laingiidae & Fabienna sphaerica & AY920797 & AY920767 & AM183133 & MHNG INVE 33453 \\
\hline Proboscidactylidae & Proboscidactyla flavicirrata & $\mathrm{EU}_{305527}$ & $\mathrm{EU}_{305500}$ & $\mathrm{EU}_{305480}$ & USNM 1074994 \\
\hline Proboscidactylidae & Proboscidactyla ornata & $\mathrm{EU}_{272587}$ & $\mathrm{EU}_{272631}$ & $\mathrm{EU}_{305481}$ & KUNHM 2767 \\
\hline Ptilocodiidae & Hydrichthella epigorgia & $\mathrm{EU}_{272569}$ & $\mathrm{EU}_{272622}$ & $\mathrm{EU}_{305478}$ & KUNHM 2665 \\
\hline \multicolumn{6}{|l|}{ Filifera III } \\
\hline Hydractiniidae & Clava multicornis & $\mathrm{EU}_{272552}$ & $\mathrm{EU}_{272609}$ & $\mathrm{EU}_{305471}$ & \\
\hline Hydractiniidae & Clavactinia gallensis & $\mathrm{EU}_{272553}$ & $\mathrm{EU}_{272610}$ & $\mathrm{EU}_{448101}$ & MHNG INVE 33470 \\
\hline Hydractiniidae & Hydractinia sp. & $\mathrm{EU}_{305518}$ & $\mathrm{EU}_{305495}$ & $\mathrm{EU}_{305477}$ & KUNHM 2876 \\
\hline Hydractiniidae & Hydractinia symbiolongicarpus & $\mathrm{EU}_{272568}$ & $\mathrm{EU}_{272621}$ & & \\
\hline Hydractiniidae & Podocoryne carnea & $\mathrm{AY}_{920802}$ & $\mathrm{AF}_{358092}$ & $\mathrm{AY}_{512513}$ & \\
\hline Stylasteridae & Adelopora crassilabrum & $\mathrm{EU}_{272541}$ & $\mathrm{EU}_{272642}$ & & USNM 1027760 \\
\hline Stylasteridae & Conopora anthohelia & $\mathrm{EU}_{305509}$ & & & \\
\hline Stylasteridae & Crypthelia cryptotrema & $\mathrm{EU}_{272558}$ & $\mathrm{EU}_{272641}$ & & USNM 1027758 \\
\hline Stylasteridae & Lepidopora microstylus & $\mathrm{EU}_{272572}$ & $\mathrm{EU}_{272644}$ & & USNM 1027724 \\
\hline Stylasteridae & Pseudocrypthelia pachypoma & $\mathrm{EU}_{272589}$ & $\mathrm{EU}_{272643}$ & & USNM 1027728 \\
\hline \multicolumn{6}{|l|}{ Filifera IV } \\
\hline Bougainvilliidae & Dicoryne conybearei & $\mathrm{EU}_{272559}$ & $\mathrm{EU}_{272614}$ & AM183141 & MHNG INVE 32949 \\
\hline Bougainvilliidae & Bimeria vestita & $\mathrm{EU}_{272548}$ & $\mathrm{EU}_{272605}$ & AM183130 & \\
\hline Bougainvilliidae & Bougainvillia carolinensis & $\mathrm{EU}_{272549}$ & $\mathrm{EU}_{272606}$ & & \\
\hline Bougainvilliidae & Bougainvillia fulva & $\mathrm{EU}_{305507}$ & $\mathrm{EU}_{305490}$ & $\mathrm{EU}_{305470}$ & KUNHM 2816 \\
\hline Bougainvilliidae & Garveia annulata/Garveia sp. & $\mathrm{EU}_{272564}$ & AY920766 & & KUNHM 2860 \\
\hline Bougainvilliidae & Koellikerina fasciculata & $\mathrm{EU}_{272571}$ & $\mathrm{EU}_{272623}$ & AM183129 & \\
\hline Bougainvilliidae & Pachycordyle pusilla & $\mathrm{EU}_{272579}$ & $\mathrm{EU}_{272627}$ & AM183132 & MHNG INVE 32953 \\
\hline Bougainvilliidae & Pruvotella (Garvia) grisea & $\mathrm{EU}_{272588}$ & $\mathrm{EU}_{272632}$ & AM183131 & MHNG INVE 34436 \\
\hline Oceaniidae & Cordylophora caspia & $\mathrm{EU}_{272556}$ & $\mathrm{EU}_{272612}$ & $\mathrm{EU}_{305472}$ & \\
\hline Oceaniidae & Corydendrium sp. & $\mathrm{EU}_{272557}$ & $\mathrm{EU}_{272613}$ & & KUNHM 2764 \\
\hline Oceaniidae & Rhizogeton nudus & $\mathrm{EU}_{272592}$ & $\mathrm{EU}_{272635}$ & $\mathrm{AY}_{787883}$ & MHNG INVE 35757 \\
\hline Oceaniidae & Turritopsis dohrnii & $\mathrm{EU}_{272596}$ & $\mathrm{EU}_{272638}$ & $\mathrm{AY}_{787889}$ & MHNG INVE 29753 \\
\hline Oceaniidae & Turritopsis nutricula & $\mathrm{EU}_{305538}$ & $\mathrm{EU}_{305504}$ & $\mathrm{EU}_{305486}$ & KUNHM 2817 \\
\hline Pandeidae & Hydrichthys boycei & $\mathrm{EU}_{272570}$ & $\mathrm{EU}_{305496}$ & EU448102 & MHNG INVE 37417 \\
\hline Pandeidae & Leuckartiara octona & $\mathrm{EU}_{272573}$ & $\mathrm{EU}_{272624}$ & $\mathrm{AM}_{411421}$ & \\
\hline Pandeidae & Neoturris breviconis & $\mathrm{EU}_{305524}$ & EU448097 & EU448103 & KUNHM 002961 \\
\hline Pandeidae & Pandea sp. & $\mathrm{EU}_{272580}$ & AY920765 & & \\
\hline Rathkeidae & Lizzia blondina & $\mathrm{EU}_{272574}$ & $\mathrm{EU}_{272625}$ & $\mathrm{AM}_{411417}$ & \\
\hline Rathkeidae & Rathkea octopunctata & $\mathrm{EU}_{272591}$ & $\mathrm{EU}_{272634}$ & $\mathrm{EU}_{305483}$ & KUMIP 314321 \\
\hline \multicolumn{6}{|l|}{ Leptothecata } \\
\hline \multicolumn{6}{|l|}{ Conica } \\
\hline Aequoreidae & Aequorea aequorea & $\mathrm{EU}_{305505}$ & $\mathrm{AF}_{358076}$ & AY512518 & \\
\hline Aequoreidae & Aequorea floridana & $\mathrm{EU}_{305506}$ & & & USNHM PENDING \\
\hline
\end{tabular}


Table 1. Continued

\begin{tabular}{|c|c|c|c|c|c|}
\hline \multicolumn{2}{|l|}{ Taxonomic hierarchy } & \multirow{2}{*}{$\begin{array}{l}\mathbf{2 8 S} \\
\text { AY920799 }\end{array}$} & \multirow{2}{*}{$\frac{\mathbf{1 8 S}}{\mathrm{AF}_{358077}}$} & \multirow{2}{*}{$\begin{array}{l}16 \mathrm{~S} \\
\mathrm{EU}_{305469}\end{array}$} & \multirow{2}{*}{$\begin{array}{l}\text { Voucher } \\
\text { KUNHM } 2867\end{array}$} \\
\hline Aequoreidae & Aequorea victoria & & & & \\
\hline Aequoreidae & Rhacostoma atlantica & $\mathrm{EU}_{305528}$ & $\mathrm{EU}_{305501}$ & & \\
\hline Aglaopheniidae & Aglaophenia tubiformis & $\mathrm{EU}_{272543}$ & $\mathrm{EU}_{272601}$ & AY787914 & MHNG INVE 29967 \\
\hline Blackfordiidae & Blackfordia virginica & AY920800 & $\mathrm{AF}_{358078}$ & $\mathrm{AY}_{512516}$ & \\
\hline Eirenidae & Eutima sapinhoa & $\mathrm{EU}_{305515}$ & $\mathrm{EU}_{305493}$ & & \\
\hline Haleciidae & Halecium muricatum & $\mathrm{EU}_{272565}$ & $\mathrm{EU}_{272619}$ & $\mathrm{AY}_{787915}$ & MHNG INVE 29028 \\
\hline Halopterididae & Halopteris minuta & $\mathrm{EU}_{272567}$ & $\mathrm{EU}_{272620}$ & $\mathrm{AY} 787912$ & MHNG INVE 25073 \\
\hline Halopterididae & Monostaechas quadridens & $E_{305521}$ & $\mathrm{EU}_{305497}$ & DQ855941 & \\
\hline Hebellidae & Anthohebella parasitica & $\mathrm{EU}_{272545}$ & $\mathrm{EU}_{272603}$ & $\mathrm{AY} 787918$ & MHNG INVE 29762 \\
\hline Lafoeidae & Lafoea dumosa & $\mathrm{EU}_{305520}$ & & $\mathrm{AY} 787917$ & MHNG INVE 29952 \\
\hline Laodiceidae & Melicertissa sp. & AY920798 & $\mathrm{AF}_{358075}$ & $\mathrm{AY}_{512515}$ & \\
\hline Malagazziidae & Octophialucium indicum & $\mathrm{EU}_{272577}$ & $\mathrm{EU}_{272626}$ & AY787897 & MHNG INVE 29970 \\
\hline Melicertidae & Melicertum octocostatum & $\mathrm{EU}_{272575}$ & AY920757 & $E_{305479}$ & USNM 1073342 \\
\hline Mitrocomidae & Tiaropsidium kelseyi & $\mathrm{EU}_{305537}$ & $\mathrm{AF}_{358079}$ & $\mathrm{EU}_{305485}$ & \\
\hline Plumulariidae & Nemertesia antennina & $\mathrm{EU}_{305523}$ & $\mathrm{EU}_{305498}$ & AY787910 & MHNG INVE 29954 \\
\hline Plumulariidae & Plumularia hyalina & $\mathrm{EU}_{305525}$ & $\mathrm{EU}_{305499}$ & $\mathrm{AY} 787913$ & MHNG INVE 25333 \\
\hline Sertulariidae & Abietinaria filicula & $\mathrm{EU}_{272540}$ & $\mathrm{EU}_{272600}$ & $\mathrm{AY} 787899$ & MHNG INVE 29947 \\
\hline Sertulariidae & Diphasia fallax & $\mathrm{EU}_{305511}$ & $E_{305491}$ & AY787901 & MHNG INVE 29950 \\
\hline Sertulariidae & Hydrallmania falcata & $\mathrm{EU}_{305519}$ & & $\mathrm{AY} 787900$ & MHNG INVE 29948 \\
\hline Sertulariidae & Sertularia cupressina & $\mathrm{EU}_{305531}$ & & $\mathrm{AY} 787905$ & MHNG INVE 29949 \\
\hline Sertulariidae & Sertularia perpusilla & $\mathrm{EU}_{305532}$ & & $\mathrm{AY} 787894$ & MHNG INVE 29765 \\
\hline Sertulariidae & Thuiaria thuja & $\mathrm{EU}_{305536}$ & $\mathrm{EU}_{305503}$ & AY787908 & MHNG INVE 29951 \\
\hline \multicolumn{6}{|l|}{ Proboscidoidea } \\
\hline Campanulariidae & Clytia noliformis & $\mathrm{EU}_{272554}$ & $\mathrm{EU}_{272611}$ & DQ064792 & \\
\hline \multicolumn{6}{|l|}{ Siphonophorae } \\
\hline \multicolumn{6}{|l|}{ Calycophorae } \\
\hline Clausophyidae & Kephyes ovata & $\mathrm{EU}_{305508}$ & $\mathrm{AY}_{937336}$ & AY935294 & YPM 35349 \\
\hline Diphyidae & Sulculeolaria quadrivalvis & $\mathrm{EU}_{272594}$ & $\mathrm{AY}_{937353}$ & AY935311 & YPM 35357 \\
\hline Hippopodiidae & Hippopodius hippopus & $\mathrm{EU}_{305517}$ & AY937341 & AY935314 & YPM 35045 \\
\hline Prayidae & Nectadamas diomedeae & $\mathrm{EU}_{305522}$ & AY937348 & AY935306 & YPM 35352 \\
\hline Prayidae & Nectopyramis sp./N. natans & AY026377 & $\mathrm{AF}_{358068}$ & AY935307 & \\
\hline Prayidae & Praya dubia & $\mathrm{EU}_{305526}$ & AY937326 & AY935285 & YPM 35346 \\
\hline Prayidae & Rosacea flaccida & $\mathrm{EU}_{305529}$ & AY937328 & & YPM 35041 \\
\hline \multicolumn{6}{|l|}{ Physonectae } \\
\hline Agalmatidae & Agalma elegans & $\mathrm{EU}_{272542}$ & AY937313 & AY935271 & YPM 35029 \\
\hline Agalmatidae & Cordagalma cordiforme & $\mathrm{EU}_{272555}$ & AY937317 & AY935275 & YPM 35032 \\
\hline Agalmatidae & Halistemma rubrum & $\mathrm{EU}_{272566}$ & AY937358 & AY935316 & YPM 35359 \\
\hline Agalmatidae & Nanomia bijuga & $\mathrm{EU}_{272576}$ & AY937338 & AY935296 & YPM 35043 \\
\hline Agalmatidae & Stephanomia amphytridis & $\mathrm{EU}_{305535}$ & AY937322 & $\mathrm{AY}_{935280}$ & YPM 35076 \\
\hline Apolemiidae & Apolemia sp. & $\mathrm{EU}_{272546}$ & AY937331 & AY935290 & YPM 35090 \\
\hline Erennidae & Erenna sp. & $\mathrm{EU}_{305512}$ & AY937361 & AY935319 & YPM 35362 \\
\hline Forskaliidae & Forskalia edwardsi & $\mathrm{EU}_{305516}$ & AY937320 & AY935278 & YPM 35036 \\
\hline Physophoridae & Physophora hydrostatica & $\mathrm{EU}_{272582}$ & AY937342 & AY935300 & YPM 35046 \\
\hline Rhodaliidae & Stephalia dilata & $\mathrm{EU}_{305534}$ & AY937357 & AY935315 & YPM 35358 \\
\hline \multicolumn{6}{|l|}{ Cystonectae } \\
\hline Physaliidae & Physalia physalis & $\mathrm{EU}_{448095}$ & $\mathrm{AY}_{358065}$ & AY935284 & YPM 35345 \\
\hline \multicolumn{6}{|l|}{ Trachylina } \\
\hline Aeginidae & Aegina citrea & AY920789 & $\mathrm{AF}_{358058}$ & EU293997 & \\
\hline Cuninidae & Solmissus marshalli & AY920790 & $\mathrm{AF}_{358060}$ & & \\
\hline Cuninidae & Solmundella bitentaculata & $\mathrm{EU}_{247797}$ & $\mathrm{EU}_{247812}$ & & MHNG 31746 \\
\hline Cuninidae & Solmundella bitentaculata & & & EU293998 & USNM 1107456 \\
\hline Halicreatidae & Haliscera conica & EU247797 & $\mathrm{AF}_{358064}$ & $\mathrm{EU}_{293981}$ & \\
\hline Oliandiasidae & Limnocnida tanganyicae & AY920795 & AY920755 & $\mathrm{EU}_{293972}$ & USNM 1075114 \\
\hline Oliandiasidae & Aglauropsis aeora & AY920793 & AY920754 & $\mathrm{EU}_{293973}$ & USNM 1073327 \\
\hline Oliandiasidae & Astrohydra japonica & AY920794 & & EU293975 & \\
\hline Oliandiasidae & Olindias sambaquiensis & $\mathrm{EU}_{247809}$ & $\mathrm{EU}_{247814}$ & & \\
\hline Rhopalonematidae & Aglantha digitale & AY920791 & $\mathrm{EU}_{247821}$ & $\mathrm{EU}_{293985}$ & USNM 1073329 \\
\hline Rhopalonematidae & Aglaura hemistoma & $\mathrm{EU}_{247803}$ & $\mathrm{EU}_{247818}$ & & MHNG 31745 \\
\hline Rhopalonematidae & Aglaura hemistoma & & & EU293984 & KUMIP 314322 \\
\hline Rhopalonematidae & Pantachogon haeckeli & $\mathrm{AY}_{920792}$ & $\mathrm{AF}_{358062}$ & & \\
\hline Rhopalonematidae & Pantachogon haeckeli & & & $\mathrm{EU}_{293988}$ & USNM 111078 \\
\hline Rhopalonematidae & Rhopalonema velatum & $\mathrm{EU}_{247804}$ & $\mathrm{EU}_{247819}$ & EU293992 & \\
\hline Tetraplatiidae & Tetraplatia volitans & DQ002502 & DQ002501 & $\mathrm{EU}_{293999}$ & KUMIP 314322 \\
\hline
\end{tabular}

KUMIP, University of Kansas Museum of Invertebrate Paleontology; KUNHM, University of Kansas Natural History Museum; MHNG, Muséum d'Histoire Naturelle de Genève; YPM, Yale-Peabody Museum; USNM, US National Museum of Natural History. 
Table 2. Summary of genetic markers used in this study.

\begin{tabular}{llll}
\hline Marker & Primer source & Length after gblocks (bp) (\% retained) & No. of parsimony informative characters (\% informative) \\
\hline 28 S & Evans et al., 2008 & $2959(81 \%)$ & $969(33 \%)$ \\
$18 \mathrm{~S}$ & Medina et al., 2001 & $1648(82 \%)$ & $407(25 \%)$ \\
$16 \mathrm{~S}$ & Cunningham \& Buss, 1993 & $439(55 \%)$ & $323(74 \%)$ \\
\hline
\end{tabular}

major clades. Given the inconclusiveness of these results, any discussion of relationships between major hydroidolinan clades would be premature. By contrast, within each of the major clades, the topologies in the ML and MP analyses are largely congruent and most of the nodes within these clades display high bootstrap support (Figures 1 \& 2). Thus we focus our discussion below on the composition and relationships within these clades.

\section{Capitata sensu stricto}

Capitata is traditionally defined by the presence of capitate tentacles at some stage in its life cycle (Reese, 1957; Petersen, 1990). Recent molecular phylogenetic analyses have questioned the monophyly of Capitata and instead suggest that there are two clades, Aplanulata (sensu Collins et al., 2005) and non-Aplanulata capitates (Collins, 2002; Collins et al., 2005, 2006). Our ML and MP analyses provide strong support (bootstrap values $=100$ and 96 respectively) for a clade of capitates to the exclusion of aplanulata taxa. We refer to this clade as Capitata sensu stricto herein. Within Capitata sensu stricto the topologies between the ML and MP analyses are nearly identical (Figures 1 \& 2 ). Both optimality criteria indicate support for the suborder Zancleida including Cladocorynidae, Porpitidae and Zancleidae (sensu Peterson, 1990), but also including Solanderiidae. Moerisia and Pennaria together form a sister taxon to the Zancleida clade (MP; Figure 2) or as successive sister taxa (ML; Figure 1). A Corynidae + Polyorchidae clade is strongly supported under both optimality criteria (Figures 1 \& 2). These topologies are largely consistent with that of Collins et al. (2005, 2006).

\section{Aplanulata}

Aplanulata (Collins et al., 2005) is a clade supported by previous molecular phylogenetic analyses (Collins et al., 2006) and is united by the lack of a ciliated planula stage (Petersen, 1990). Our analyses of Corymorphidae, Hydridae, Candelabridae and Tubulariidae representatives provide strong support for the monophyly of Aplanulata (bootstrap values $=100$ for ML and MP) (Figures 1 \& 2). Although our sampling is limited, within Aplanulata, there is strong support and nearly complete congruence between ML and MP topologies and these relationships are largely consistent with that recovered from Collins et al. (2005) that used partial $16 \mathrm{~S}$ data. Corymorphidae and Tubulariidae are both monophyletic and there is strong support for a Corymorphidae + Tubulariidae clade (bootstrap values $=100$ for ML and MP). The Hydra + Candelabrum clade is the sister group to the rest of Aplanulata in the MP analysis (Figure 2) but are successive sister taxa in the ML analysis (Figure 1). As discussed in Collins et al. (2006), there are other putative Aplanulata families that await future sampling and analyses.

\section{Filifera I: Eudendriidae}

Our MP and ML analyses provide strong support for a Eudendriidae clade (bootstrap values $=98$ for $\mathrm{ML}$ and 85 for MP), apart from other filiferan clades (Figures 1 \& 2 ). Eudendriidae as a clade distinct from other filiferans is supported by many synapomorphies including the absence of desmoneme nematocysts, a styloid-shaped gonophore and a trumpet-shaped hypostome (Marques, 1996). Because of these unique traits, a possible sister-group relationship of the Eudendriidae with other filiferans remains dubious (Marques, 1996, 2001b).

\section{Filifera II: Fabienna/Proboscidactyla/ Brinckmannia/Hydrichthella}

The monophyly of Fabienna + Proboscidactyla + Brinkmannia is well supported (bootstrap values $=94$ for ML and 79 for MP) but the node that includes Hydrichthella as its sister taxon has relatively low support (bootstrap values $=57$ for ML and MP). An association between the Laingiomedusae Fabienna and Proboscidactylidae is supported by morphological evidence including a solid ring canal and macrobasic euryteles (Schuchert, 1996). A previous molecular analysis supported this relationship (Collins et al., 2006). In addition, there are a number of morphological features that support the association of Fabienna/Proboscidactyla with Brinckmannia (Filifera incertae sedis) and the ptilocodiid Hydrichthella. Schuchert \& Reiswig (2006) argued for a close relationship between Brinckmannia and Proboscidactylidae based on the shape of their hydranths and $16 \mathrm{~S}$ sequence similarity. Although the polyp stage in Fabienna is unknown, the other taxa share a synapomorphy of hydranths with reduced tentacles: in Brinckmannia, hydranths have no tentacles (Schuchert \& Reiswig, 2006), Hydrichthella has no tentacles on its gastrozooids (dactylozooids have many tentacles) and Proboscidactyla has only two tentacles on its hydranths. Interestingly, many of the species in this group are closely associated with another invertebrate as a substrate: Hydrichthella is found on an octocoral, Brinckmannia within the tissues of a hexactinellid sponge and Proboscidatyla on tubes of sabellid polychaetes. In addition, although the ptilocodiid Hydrichthella does not have a medusa, the medusae of Fabienna are strikingly similar to that of another ptilocodiid species, Thecocodium quadratum (Collins et al., 2006).

\section{Filifera III: Hydractiniidae/Stylasteridae}

Our ML and MP analyses are congruent in identifying a clade that includes Hydractiniidae and Stylasteridae (bootstrap values $=86$ for $\mathrm{ML}$ and $<50$ for $\mathrm{MP}$ ). There is strong support for monophyly of Stylasteridae (bootstrap values $=100$ for $\mathrm{ML}$ and 99 for MP) (Figures 1 \& 2 ). 


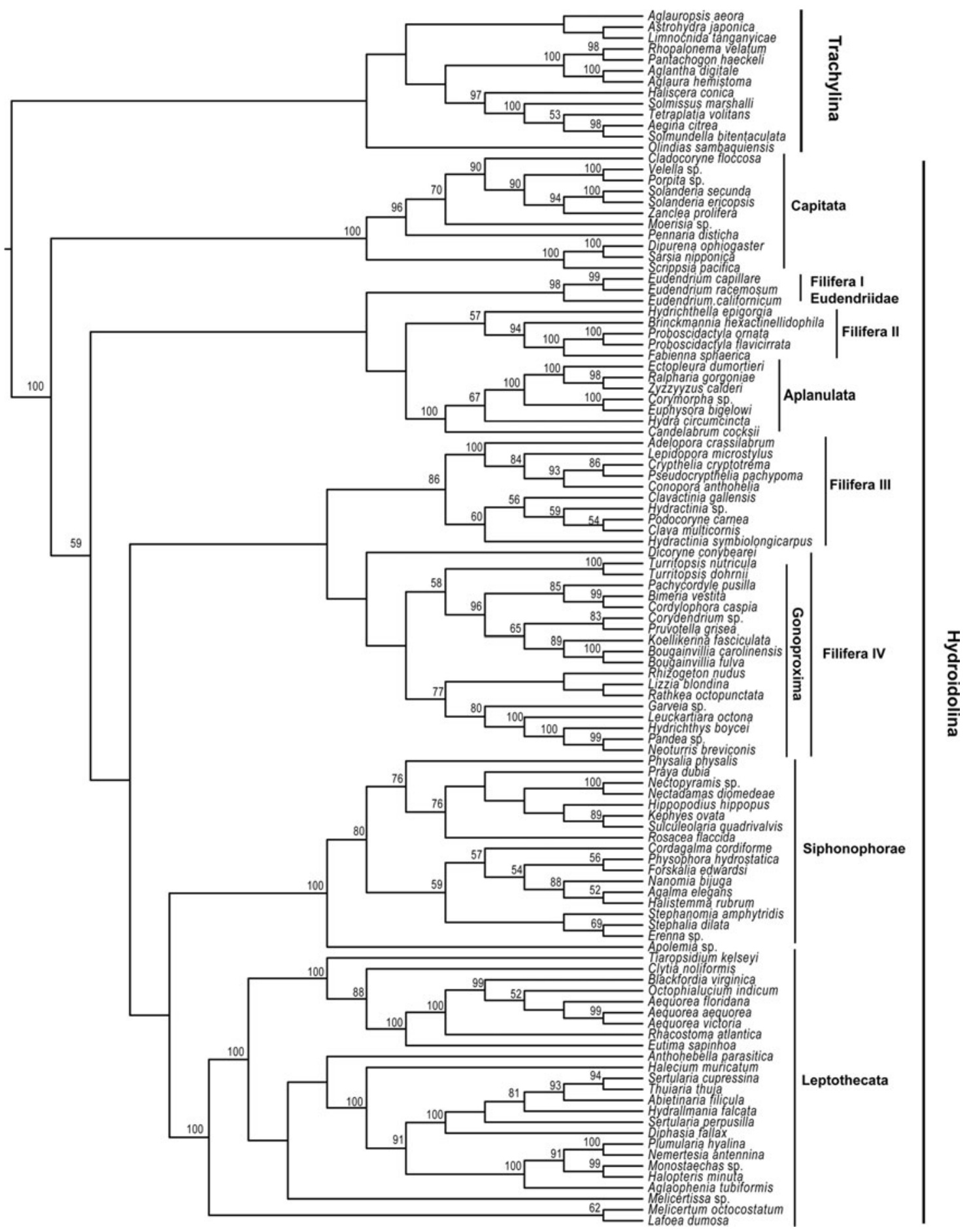

Fig. 1. Phylogenetic hypothesis among 110 hydrozoan taxa, based on a maximum likelihood criterion of a combined dataset of nearly complete $28 \mathrm{~S}$, nearly complete $18 \mathrm{~S}$ and partial $16 \mathrm{~S}$ rDNA sequences. Bootstrap values greater that 50 are indicated above nodes. The assumed model (GTR $+\mathrm{I}+\mathrm{G})$ with six substitutions rates estimated from the data (A-C, 0.8735; A-G, 2.9730; A-T, 1.6586; C-G, 0.8463; C-T, 5.2641; G-T, 1.00oo), an assumed proportion of invariant sites (0.5740) and a gamma shaped parameter of (0.6021).

Although the hydractiniids are monophyletic in the ML analysis (bootstrap value $=60$ ) (Figure 1 ) the MP analysis places them as paraphyletic relative to the stylasterids (Figure 2). Our analyses show Clava multicornis as the sister taxon to the hydractiniid Podocoryne carnea with strong support in both ML and MP trees (bootstrap values $=94$ for ML and 89 for MP). Clava has traditionally been placed in the family Clavidae, although Schuchert
(2001) argued, based on the similarities of Clava to other hydractiniids (Bouillon et al., 1997), that the genus Clava should be moved to the hydractiniids and the other Clavidae genera moved to the nominal family Oceaniidae (Schuchert, 2004). Our analysis supports the interpretation that Clava is a hydractiniid.

The close relationship between the Hydractiniidae and Stylasteridae families has previously been suggested based 


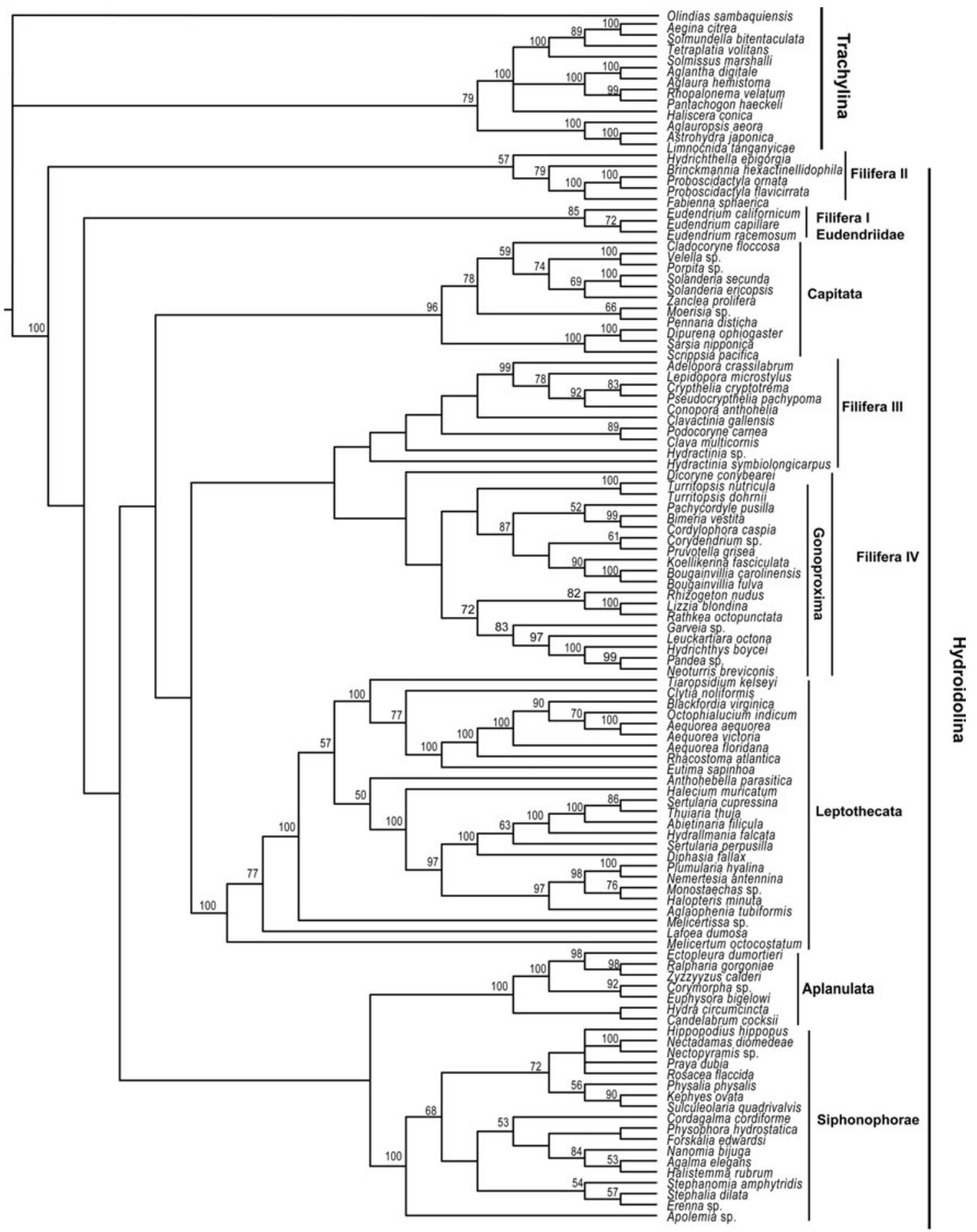

Fig. 2. Phylogenetic hypothesis among 110 hydrozoan taxa, based on parsimony criterion of a combined dataset of nearly complete $28 \mathrm{~S}$, nearly complete $18 \mathrm{~S}$ and partial $16 \mathrm{~S}$ rDNA sequences. Bootstrap values greater that 50 are indicated above nodes. Topology is a strict consensus of the 10 most parsimonious trees (5046 characters). Length: 13246 steps, CI-0.26; RI-0.59.

on a number of synapomorphies including polymorphic polyps and the perisarc or skeleton covered stolons (Bouillon, 1978; Petersen, 1979). Bouillon (1978) placed these families in the superfamily Hydractinoidea, which also includes Ptilocodiidae, Rathkeidae and Rhysiidae. Though we did not sample any members of Rhysiidae, our analyses do not support Hydractinoidea, as sampled Ptilocodiidae and Rathkeidae members are placed outside this clade.

\section{Filifera IV: Gonoproxima + Dicoryne- Bougainvilliidae/Oceaniidae/Pandeidae/ Rathkeidae}

Our ML and MP analyses support the monophyly of a clade that includes representatives of Bougainvilliidae, Oceaniidae, Pandeidae and Rathkeidae. This clade has relatively weak support (bootstrap values $<50$ for $\mathrm{ML}$ and $\mathrm{MP}$ ) and must be viewed as tentative. Within the clade the topologies are 
congruent between the ML and MP analyses. Of the four families, Pandeidae and Rathkeidae are monophyletic, whereas Bougainvilliidae and Oceaniidae are polyphyletic. Although association of these four families is somewhat surprising, they all share a striking synapomorphy. The species in all four families bear gonophores on hydrocauli, pedicels, or stolons and not on the hydranth body. The shift of the gonophores from the hydranth body to the region below is an apomorphy (Schuchert, 2001). Backed by this synapomorphy, we name this clade Gonoproxima. The 'bougainvilliid' Dicoryne, which is distinct from other bougainviliid species in that it produces gonophores on blastostyles, is placed as the sister taxon to Gonoproxima in both the ML and MP analyses (Figures 1 \& 2). Interestingly, many taxa included in this group have perisarc extending over the hydranth body, either as a gelatinous structure or pseudohydrotheca.

Two species within Gonoproxima, Cordylophora caspia (sampled here) and Pachycordyle kubotai (not sampled) live in fresh water. In our analyses, Cordylophora and Pachycordyle, which also contains brackish and marine species, are indicated to be close relatives under both optimality criteria, forming a clade with the bougainvilliid Bimeria. With denser taxon sampling within Gonoproxima and more targeted phylogenetic analyses, it should be possible to ascertain whether the fresh water habit was evolved one or more times in this clade and potentially whether freshwater species are descended from ancestors that lived in brackish environments.

\section{Siphonophorae}

The siphonophores have historically been split into three major groups, Cystonectae, Physonectae and Calycophorae. Collins (2002) placed the cystonect Physalia as sister to the other included siphonophores and suggested that Physonectae may be paraphyletic with respect to the Calycophorae. A later study (Dunn et al., 2005), that considered additional taxa and two genes (18S and 16S) found that cystonects form a monophyletic group that is sister to the remaining siphonophores and the paraphyly of Physonectae was recovered with significant support. Dunn et al. (2005) erected the name Codonophora to refer to the clade comprising taxa assigned to Physonectae and Calycophorae (i.e. the clade that is sister to Cystonectae).

Our ML and MP analyses are consistent with the findings of Collins (2002) and Dunn et al. (2005) in that Siphonophorae is a strongly supported monophyletic group (bootstrap values = 100 for ML and MP). Our ML and MP analysis also recovered Physonectae as paraphyletic and Calycophorae derived within this clade (Figures 1 \& 2 ). We did not however find support for Codonophora (sensu Dunn et al., 2005). Our ML analysis placed the cystonect Physalia as the sister taxon to Calycophorae (Figure 1), not as the earliest diverging member of siphonophores (Collins 2002; Dunn et al., 2005). The MP analysis also recovered a probable paraphyletic Physonectae, but unlike the ML analyses, Physalia was nested within Calycophorae (Figure 2). Under both optimality criteria, the physonect, Apolemia, was the earliest diverging siphonophore. Given that we have only one cystonect representative (Physalia) and that its placement is dependent on optimality criteria, we view the placement of Cystonectae relative to other siphonophores as equivocal and await further study.

\section{Leptothecata}

Our ML and MP analyses found strong support for the Leptothecata clade (bootstrap values $=100$ for ML and MP) (Figures 1 \& 2). Sampling was concentrated amongst the Conica subgroup, with the inclusion of only one Proboscoida representative, Clytia noliformis. This sampling is therefore insufficient to address the question of monophyly of its subgroups, Conica and Proboscoida.

The ML and MP topologies within Leptothecata are nearly congruent except for the placement of Lafoea relative to Melicertum (discussed below). The traditional taxonomy of Leptothecata, including the relationships of its higher groups, is largely based on similarities in the morphology of the hydrotheca and nematotheca (e.g. Bouillon, 1985, 1994). Many groups found in our analyses corroborate Bouillon's hypotheses, including the monophyly of the Plumularioidea taxa, Plumulariidae and Halopterididae, Sertulariidae and the affinities of these with Haleciidae. Recent molecular and morphological analyses also have corroborated or are consistent with these hypotheses (Leclère et al., 2007).

The affinities of the Hebellidae and Lafoeidae, based on morphological characters, were investigated by Marques et al. (2006). Although the authors hypothesized the exclusive monophyly of each family, they considered the possibility that the families are distantly related, a finding consistent with our analyses.

Campanulinida is a group of leptothecates including many diverse families: Aequoreidae, Blackfordiidae, Eirenidae, Laodiceidae, Malagazziidae, Melicertidae and Mitrocomidae. The Campanulinida taxa belonging to Aequoreidae, Blackfordiidae, Eirenidae, Malagazziidae and Mitrocomidae are a strongly supported clade that also includes Clytia noliformis (bootstrap values $=100$ for $\mathrm{ML}$ and MP). The Campanulinida belonging to Melicertidae, Melicertum octocostatum is the sister taxon to the rest of the Leptothecata in the MP analysis (Figure 2). This analysis corroborates the hypothesis of an early divergence of $M$. octocostatum (Collins et al., 2006), a species that lacks a theca but has typical leptothecate medusae. The ML analysis places Melicertum + Lafoea dumosa as the sister taxon to the rest of Leptothecata (Figure 1).

\section{CONCLUSIONS}

Anthoathecata represents a diverse order of hydroidolinans that traditionally comprises two suborders, Filifera and Capitata (reviewed in Daly et al., 2007). Although our analyses and previous molecular phylogenetic analyses (Marques \& Collins, 2000; Marques, 2001a; Collins, 2002; Collins et al., 2006; Van Iten et al., 2006) do not support the monophyly of Anthoathecata, the dissolution or re-definition of Anthoathecata is premature and should await clarification of relationships between major hydroidolinan clades. Capitata in the traditional sense comprises two clades, the Aplanulata, recognized by the lack of a free-swimming planula (Petersen, 1990) and Capitata sensu stricto. Given that there is strong support for these two groups and that there is no support for the monophyly of traditional 'Capitata' in these analyses and in previous phylogenetic analyses (Collins, 2002; Collins et al., 2005, 2006), the validity of Capitata in the traditional sense is questioned. If these clades 
are indeed separate, then Aplanulata should be referred to as its own order, separate from Capitata sensu stricto. Re-defining Capitata however, should await further clarification of Hydroidolina phylogeny. Our analyses do not support the monophyly of Filifera but this too is preliminary as the nodes separating the filiferan subgroups are weakly supported (Figures 1 \& 2).

The new augmented dataset used in our analyses provide support for four distinct filiferan clades. Notably, all of these clades possess compelling morphological synapomorphies; Gonoproxima is characterized by gonophores on regions of the colony proximal to the hydranth; Eudendriidae displays distinct polyp and hypostome morphology; the Fabiennal Proboscidactyla/Brinckmannia/Hydrichthella clade displays polyps with a reduced number of tentacles and the Hydractiniidae/Sylasteridae clade displays polymorphism. A more comprehensive sampling of hydroidolinan families should provide greater insight into these emerging patterns.

Despite increased sampling, relationships between major hydroidolinan clades remain elusive. The lack of resolution suggests that the initial radiation of Hydroidolina may have been rapid, leaving little clues regarding the sequence of hydroidolinan diversification. New molecular markers, especially if combined with other types of data, may prove helpful in resolving these deep nodes.

\section{ACKNDWLEDGEMENTS}

We would like to thank A. Lindner for DNA samples and anonymous referees for helpful comments. This study was supported from grants from NSF AToL EF-0531779 (to P.C. and A.G.C.). A.C.M. has financial support from CNPq (55.7333/2005-9, 490348/2006-8, 305735/2006-3) and FAPESP (2004/09961-4).

\section{REFERENCES}

Bouillon J. (1978) Sur un nouveau genre et une nouvelle espèce de Ptilocodidae, Hydrichtelloides reticulata et la super-famille des Hydractinoidea (Hydroida-Athecata). Steenstrupia 5, 53-67.

Bouillon J. (1985) Essai de classification des hydropolypes-hydroméduses (Hydrozoa-Cnidaria). Indo-Malayan Zoology 2, 29-243.

Bouillon J. (1994) Classe des hydrozoaires (Hydrozoa Owen, 1843). In P.P. Grassé (ed.) Traité de Zoologie T III fasc. 2 Cnidaires. Paris: Masson, pp. 29-416.

Bouillon J., Gravili C., Pagès F., Gili J.M. and Boero F. (2006) An introduction to Hydrozoa. Paris: Publications Scientifiques du Muséum, Paris.

Bouillon J., Medel D. and Peña Cantero A.L. (1997) The taxonomic status of the genus Stylactaria Stechow, 1921 (Hydroidomedusae, Anthomedusae, Hydractiniidae), with the description of a new species. Scientia Marina 61, 471-486.

Castresana J. (2000) Selection of conserved blocks from multiple alignments for their use in phylogenetic analysis. Molecular Biology and Evolution 17, 540-552.

Collins A.G. (2002) Phylogeny of Medusozoa and the evolution of cnidarian life cycles. Journal of Evolutionary Biology 15, 418-432.

Collins A.G., Schuchert P., Marques A.C., Jankowski T., Medina M. and Schierwater B. (2006) Medusozoan phylogeny and character evolution clarified by new large and small subunit rDNA data and an assessment of the utility of phylogenetic mixture models. Systematic Biology 55, 97-115.

Collins A.G., Winkelmann S., Hadrys H. and Schierwater B. (2005) Phylogeny of Capitata and Corynidae (Cnidarian, Hydrozoa) in light of mitochondrial 16S rDNA data. Zoologica Scripta 34, 91-99.

Cunningham C.W. and Buss L.W. (1993) Molecular evidence for multiple episodes of paedomorphosis in the family Hydractiniidae. Biochemical Systematics and Ecolgy 21, 57-69.

Daly M., Brugler M.R., Cartwright P., Collins A.G., Dawson M.N., Fautin D.G., France S.C., McFadden C.S., Opresko D.M., Rodrigues E., Romano S.L. and Stake J.L. (2007) The phylum Cnidaria: a review of phylogenetic patterns and diversity 300 years after Linnaeus. Zootaxa 1668, 127-182.

Dunn C.W., Pugh P.R. and Haddock S.H.D. (2005) Molecular phylogenetics of siphonophora (Cnidaria), with implications for the evolution of functional specialization. Systematic Biology 54, 916-935.

Evans N.M., Lindner A., Raikova E.V., Collins A.G. and Cartwright P. (2008) Phylogenetic placement of the enigmatic parasite, Polypodium hydriforme, within the Phylum Cnidaria. BMC Evolutionary Biology 8, 139.

Edgar R.C. (2004) MUSCLE: multiple sequence alignment with high accuracy and high throughput. Nucleic Acids Research 32, $1792-1797$.

Govindarajan A.F., Boero F. and Halanych K.M. (2006) Phylogenetic analysis with multiple markers indicates repeated loss of the adult medusa stage in Campanulariidae (Hydrozoa, Cnidaria). Molecular Phylogenetics and Evolution 38, 820-834.

Leclère L., Schuchert P. and Manuel M. (2007) Phylogeny of the Plumularioidea (Hydrozoa, Leptothecata): evolution of colonial organisation and life cycle. Zoologica Scripta 36, 371-394.

Marques A.C. (1996) A critical analysis of a cladistic study of the genus Eudendrium (Cnidaria: Hydrozoa), with some comments on the family Eudendriidae. Journal of Comparative Biology 1, 153-162.

Marques A.C. (2001a) Simplifying hydrozoan classification: inappropriateness of the group Hydroidomedusae in a phylogenetic context. Contributions to Zoology 70, 175-179.

Marques A.C. (2001b) O gênero Eudendrium (Cnidaria, Hydrozoa, Anthomedusae) no Brasil. Papéis Avulsos de Zoologia 41, 329-405.

Marques A.C. and Collins A.G. (2004) Cladistic analysis of Medusozoa and cnidarian evolution. Invertebrate Biology 123, 23-42.

Marques A.C. and Migotto A.E. (2001) Cladistic analysis and new classification of the family Tubulariidae (Hydrozoa, Anthomedusae). Papéis Avulsos de Zoologia 41, 465-488.

Marques A.C., Peña Cantero A.L. and Migotto A.E. (2006) An overview of the phylogeny of the families Lafoeidae and Hebellidae (Hydrozoa: Leptothecata): their composition and classification. Invertebrate Systematics 20, 43-58.

Medina M., Collins A.G., Silberman J.D. and Sogin M.L. (2001) Evaluating hypotheses of basal animal phylogeny using complete sequences of large and small subunit rRNA. Proceedings of the National Academy of Science of the USA 98, 9707-9712.

Medlin L.H., Elwood H.J., Stickel S. and Sogin M.L. (1988) The characterization of enzymatically amplified eukaryotic 16 S-like rRNA-coding regions. Gene 71, 491-499.

Peña Cantero A.L. and Marques A.C. (1999) Phylogenetic analysis of the Antarctic genus Oswaldella Stechow, 1919 (Hydrozoa, Leptomedusae, Kirchenpaueriidae). Contributions to Zoology 68, 83-93.

Petersen K.W. (1979) Development of coloniality in Hydrozoa. In G. Larwood and B.R. Rosen (eds) Biology and systematics of colonial organisms. London: Academic Press, pp. 105-139. 
Petersen K.W. (1990) Evolution and taxonomy in capitate hydroids and medusae (Cnidaria: Hydrozoa). Zoological Journal of the Linnean Society 100, 1-231.

Posada D. and Crandall K.A. (2000) Modeltest: testing the model of DNA substitution. Bioinformatics 14, 817-818.

Rees W.J. (1957) Evolutionary trends in the classification of capitate hydroids and medusae. Bulletin of the British Museum (Natural History) Zoology 4, 455-534.

Schuchert P. (1996) The marine fauna of New Zealand: athecate hydroids and their medusae (Cnidaria: Hydrozoa). New Zealand Oceanographic Institute Memoir 106, 1-159.

Schuchert P. (2001) Hydroids of Greenland and Iceland (Cnidaria, Hydrozoa). Meddelelser om Grønland, Bioscience 53, 1-184.

Schuchert P. (2004) Revision of the European athecate hydroids and their medusae (Hydrozoa, Cnidaria): families of Oceanidae and Pachycordylidae. Revue Suisse de Zoologie 111, 315-369.

Schuchert P. and Reiswig H.M. (2006) Brinckmannia hexactinellidophila, n. gen., n. sp.: a hydroid living in tissues of glass sponges of the reefs, fjords, and seamounts of Pacific Canada and Alaska. Canadian Journal of Zoology 84, 564-572.

Swofford D.L. (1998) PAUP*_Phylogenetic Analysis Using Parsimony (*and other methods). Sunderland, MA: Sinauer.

Van Iten H., Leme J.M., Simões M.G., Marques A.C. and Collins A.G. (2006) Reassessment of the phylogenetic position of conulariids (?Ediacaran-Triassic) within the subphylum Medusozoa (Phylum Cnidaria). Journal of Systematic Paleontology 4, 109-118.

and

Zwickl D.J. (2006) Genetic algorithm approaches for the phylogenetic analysis of large biological sequence datasets under the maximum likelihood criterion. PhD thesis. Austin: The University of Texas.

\section{Correspondence should be addressed to:}

Paulyn Cartwright

Department of Ecology and Evolutionary Biology University of Kansas, Lawrence, KS 66049, USA email: pcart@ku.edu 